\title{
$\beta$-Adrenergic receptor expression in vascular tumors
}

\author{
Karen M Chisholm ${ }^{1}$, Kay W Chang ${ }^{2}$, Mai T Truong ${ }^{2}$, Shirley Kwok ${ }^{1}$, Rob B West ${ }^{1}$ and \\ Amy E Heerema-McKenney ${ }^{1}$ \\ ${ }^{1}$ Department of Pathology, Stanford University School of Medicine, Stanford, CA, USA and ${ }^{2}$ Department of \\ Otolaryngology, Stanford University School of Medicine, Stanford, CA, USA
}

\begin{abstract}
Propranolol has recently emerged as an effective therapy for infantile hemangiomas causing regression. The $\beta$-adrenergic receptor (AR) antagonist is thought to cause vasoconstriction by its effect on nitric oxide, block angiogenesis by its effect on vascular endothelial growth factor (VEGF), and induce apoptosis. In a prior report, we identified expression of $\beta 2-A R$ (B2-AR) and its phosphorylated form (B2-ARP) in a case of infantile hemangioma that responded to propranolol treatment. We now explore the expression of $\beta A R s$ on a variety of vascular lesions utilizing a tissue microarray containing 141 lesions, including infantile hemangiomas, angiosarcomas, hemangiomas, hemangioendotheliomas, and various vascular malformations. The array was immunostained for B2-AR, B2-ARP, and B3-AR (B3-AR), and the results scored for the intensity of endothelial cell expression as negative, weak positive, or strong positive. All phases of infantile hemangiomas had strong expression of all three receptors, with the exception of only weak expression of B2-ARP in the proliferative phase infantile hemangioma. Strong expression of all three receptors was present in many hemangiomas, hemangioendotheliomas, and vascular malformations. Absent to weak expression of all three receptors was seen in glomus tumor, hobnail hemangioendothelioma, pyogenic granuloma, and reactive vascular proliferations. This is the first study to report $\beta$-AR expression in a variety of vascular lesions. Although immunohistochemical expression of the receptors does not necessarily indicate that similar pathways of responsiveness to $\beta$-blockade are present, it does raises the possibility that $\beta$-blockade could potentially affect apoptosis and decrease responsiveness to VEGF. Additional study is warranted, as therapeutic options are limited for some patients with these lesions.
\end{abstract}

Modern Pathology (2012) 25, 1446-1451; doi:10.1038/modpathol.2012.108; published online 29 June 2012

Keywords: $\beta$-adrenergic; immunohistochemistry; infantile hemangiomas; tissue microarray; vascular

Infantile hemangiomas are the most common tumors of infancy. ${ }^{1}$ They typically appear in the first weeks of life in a rapid growth phase, proliferate over the course of 3-6 months, stabilize over a period of few months, and then spontaneously involute over several years, usually within $5-7$ years. ${ }^{1}$ Until recently, corticosteroids had been the first-line treatment for life- or function-threatening infantile hemangiomas, with other treatment options being vincristine and interferon alpha. ${ }^{2-6}$ However, these therapies have multiple side effects, some of which are serious. ${ }^{7-9}$ In a recent case series, ${ }^{10}$ propranolol

Correspondence: Dr A Heerema-McKenney, MD, Department of Pathology, Stanford University School of Medicine, 300 Pasteur Drive, L235, Stanford, CA 94305, USA.

E-mail: aheerema@stanford.edu

Received 17 February 2012; revised 22 May 2012; accepted 22 May 2012; published online 29 June 2012 was used to treat the obstructive hypertrophic myocardiopathy caused by corticosteroid therapy for an infantile hemangioma. Unexpectedly, the propranolol caused regression of the infantile hemangioma as well. Another child who was treated with propranolol for increased cardiac output due to compression by an intracervical infantile hemangioma had reduction in the size of his infantile hemangioma after therapy began. Since then, case studies and small series of infantile hemangiomas treated successfully with propranolol or other $\beta$ blocking agents have been reported. ${ }^{11-17}$

Three different $\beta$-adrenergic receptor (AR) subtypes are known definitively to exist: $\beta 1, \beta 2$, and $\beta 3$. These $\beta$-ARs are G-protein-coupled receptors and are present on endothelial cells. When $\beta 1$ and $\beta 2$ ARs are activated, they cause vasodilation of peripheral vessels, with $\beta 2$ receptors predominating in most vascular smooth muscle, except for the 
coronary and cerebral arteries, where $\beta 1$ receptors predominate. The $\beta 3$ receptor is also identified in blood vessels as a mediator of vasodilation (reviewed in Guimaraes and Moura ${ }^{18}$ ). Mechanistically, when activated, the receptors promote intracellular signal transduction pathways through the downstream activation of cyclic adenosine monophosphate (cAMP), and hence the activation of cAMP-dependent protein kinase A (PKA). The activation of PKA leads to activation of endothelial NO synthase (eNOS), releasing nitric oxide and causing vasodilation. In addition, activation of $\beta$ ARs results in the synthesis of proangiogenic factors, such as vascular endothelial growth factor (VEGF) and basic fibroblast growth factor (bFGF), which activate proangiogenic cascades [extracellular signal-related kinases/mitogen-activated protein kinases (MAPK) cascade] promoting angiogenesis. ${ }^{19-21}$ Also, activation of $\beta$-ARs inhibits apoptosis mediated by Src tyrosine kinase, MAPK, and caspase cascades. ${ }^{20}$

PKA can phosphorylate $\beta 1$ and $\beta 2$-ARs, resulting in uncoupling and internalization. ${ }^{22}$ Dephosphorylation ensues, followed by recycling of the receptor to the cell membrane. Phosphorylation and internalization leads to desensitization. The $\beta 3-\mathrm{AR}$ is resistant to this desensitization. In addition to this difference, the $\beta 3$-AR is not blocked by the $\beta 1$ - and $\beta 2$-receptor antagonist propranolol. ${ }^{23,24}$

Propranolol, a nonselective $\beta$-AR antagonist, has been hypothesized to treat infantile hemangiomas via vasoconstriction, inhibition of angiogenesis, and induction of apoptosis. ${ }^{20}$ Propranolol-induced vasoconstriction is thought to be mediated through inhibition of eNOS and NO release, resulting in the change in color and palpable softening seen in infantile hemangiomas with the onset of propranolol treatment. ${ }^{20}$

In a prior report, we identified the immunohistochemical expression of $\beta 2-A R$ (B2-AR) and its phosphorylated form (B2-ARP) in a case of infantile hemangioma responding to propranolol treatment. ${ }^{17}$ The B2-AR demonstrated cytoplasmic staining within the endothelial cells and arteriole smooth muscle. B2-ARP demonstrated granular cytoplasmic staining, potentially representing endocytosed phosphorylated B2-AR. In this current study, we determine the expression of the B2-AR, B2-ARP, and the $\beta 3-A R$ (B3-AR) in a series of various vascular tumors on a tissue microarray, including several infantile hemangiomas in different phases of growth and involution.

\section{Materials and methods}

\section{Tissue Microarray}

The vascular tumor tissue microarray (TA 185) contained a total of $304,0.6 \mathrm{~mm}$ cores from formalin-fixed paraffin-embedded tissue, with each lesion represented in duplicate. Controls included breast ductal carcinoma, lymphoma, melanoma, skin, and placental tissue. Hematoxylin and eosin staining was used to confirm the classification of all vascular lesions according to the International Society for the Study of Vascular Anomalies. ${ }^{25}$ Overall, the microarray contained 141 lesions: angiosarcoma (47), epithelioid hemangioendothelioma (15), lymphatic vascular malformation (8), venous vascular malformation (7), littoral cell angioma (7), Kaposi sarcoma (5), intimal sarcoma (5), glomus tumor (5), spindle cell hemangioma (5), infantile hemangioma (5), epithelioid hemangioma (4), kaposiform hemangioendothelioma (4), intramuscular vascular malformation (4), hemangiopericytoma (3), arteriovenous vascular malformation (3), pyogenic granuloma (2), Masson's (2), bone hemangioma (2), and one case each of bone hemangioendothelioma, hobnail hemangioendothelioma, retiform hemangioendothelioma, synovial hemangioma, mixed small and large vessel vascular malformation, vascular malformation not otherwise specified, reactive vascular proliferation, and lymphangioleiomyomatosis.

\section{Immunohistochemical Staining}

Serial sections of $4 \mu \mathrm{m}$ were cut from the tissue microarray block and used for immunohistochemical analysis. Expression of three $\beta$-ARs: B2-AR, B2ARP, and B3-AR were evaluated for each lesion. The primary antibodies used were B2-AR ( $\beta 2$-Adrenergic Receptor antibody, Abcam, Cambridge, MA, USA; ab13300, rabbit polyclonal, 1:200 dilution, citrateinduced antigen retrieval), B2-ARP ( $\beta 2$-Adrenergic Receptor (phospho S346) antibody, Abcam;, ab62464, rabbit polyclonal, 1:200 dilution, citrateinduced antigen retrieval), and B3-AR ( $\beta 3$-Adrenergic Receptor antibody, Abcam, ab76249, 1:50 dilution, citrate-induced antigen retrieval). GLUT-1 immunohistochemical staining (GLUT1 antibody, Cell Marque, Rocklin, CA, USA; cat no. 355A-16, 1:300 dilution, citrate-induced antigen retrieval, $\mathrm{pH}$ 6.0) was used to confirm the diagnosis of the infantile hemangioma cores. The $\beta$-AR immunohistochemical reactions were visualized using the EnVision TM + system (DAKO, Carpinteria, CA, USA) with diaminobenzidine (DAB) as the substrate buffer and chromogen. The GLUT1 immunohistochemical reaction was performed on the Leica Bond Max (Bannock Burn, IL, USA) using the Bond Polymer Refine Detection system with the DAB chromogen. The intensity of endothelial cell expression was interpreted by histopathological evaluation by the primary authors (KMC and AHM), using the following criteria: $0=$ negative; $1=$ equivocal/uninterpretable; $2=$ weak positive; $3=$ strong positive. Negative scores were given to cores with $<5 \%$ staining, weak-positive scores were given to cores with staining in $<50 \%$ of the lesional cells, and strong-positive scores were given to cores with staining in $>50 \%$ of the lesional cells. In general, intensity highly correlated with percent staining 
with lesions: lesions with strong staining intensity stained $>50 \%$ of endothelial cells, whereas lesions with weaker stain intensity stained $<50 \%$ of cells. For each lesion, scores of 1 were eliminated, and the higher of the remaining scores used for analysis.

\section{Results}

\section{Immunohistochemical Scoring}

We utilized a three-tiered scale for scoring expression of B2-AR, B2-ARP, and B3-AR on the vascular tumor tissue microarray, with no staining (score 0), weakly positive staining (score 2), and strongly positive staining (score 3). Uninterpretable or equivocal stains (score 1) were also noted, and were usually due to missing cores or cores not being representative of the lesion. A total of 141 vascular lesions were represented in duplicate on the microarray. Of these 141 lesions, $80.9 \%(114 / 141)$ had an interpretable (score 0, 2, or 3) B2-AR stain, 90.1\% (127/141) B2-ARP stain, and $74.5 \%(105 / 141)$ B3-AR stain.

\section{Infantile Hemangioma Staining}

Five lesions on the tissue microarray were confirmed by GLUT-1 staining (data not shown) to be infantile hemangiomas: one in the proliferative phase, one in the late proliferative/early involutional phase, and three in the involutional stage. As shown in Figure 1, B2-AR and B3-AR stains were strongly positive in all three phases of infantile hemangiomas. The B2-ARP stain was strongly positive in the later phases of the infantile hemangiomas, but only weakly positive in the earlier proliferative phase.

\section{Vascular Lesion Staining}

A total of 125 other vascular lesions on the tissue microarray had at least one interpretable immunohistochemically stained core. Figure 2 displays examples of $\beta$-AR staining in five different vascular lesions. Table 1 records the number of lesions with interpretable staining for each $\beta$-AR and the percent of lesions that had strongly positive staining. As listed, strong expression of B2-AR was observed in multiple lesions, including all spindle cell hemangiomas, intimal sarcomas, various vascular malformations, as well as the majority of Kaposi sarcoma cases. Strong expression of B2-ARP was present in all cases of epithelioid hemangiomas, and in most epithelioid hemangioendotheliomas, kaposiform hemangioendotheliomas, littoral cell angiomas, and vascular malformations. Strong expression of B3-AR was present in the single cases of bone hemangioma,

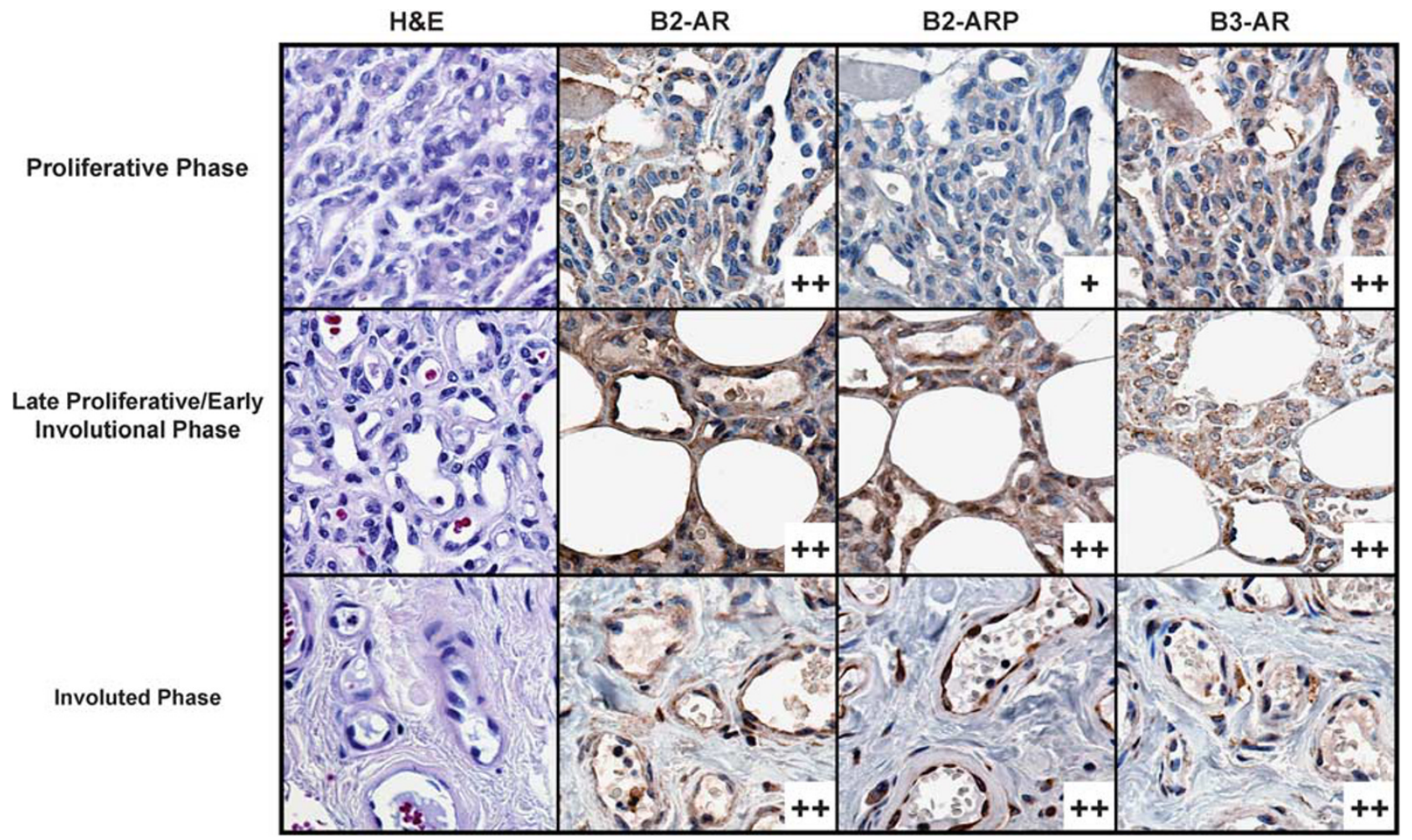

Figure $1 \beta$-Adrenergic receptor (AR) staining in infantile hemangiomas. Hematoxylin and eosin staining of three different phases of infantile hemangioma: proliferative phase, late proliferative/early involutional phase, and involuted phase was performed, followed by immunohistochemical evaluation with $\beta 2$-AR (B2-AR), its phosphorylated form (B2-ARP), and $\beta 3$ AR (B3-AR). As shown, B2-AR and B3AR, each had strong $(++)$ expression in all phases, whereas B2-ARP had weak expression in the proliferation phase $(+)$, and strong expression in the later two phases. 


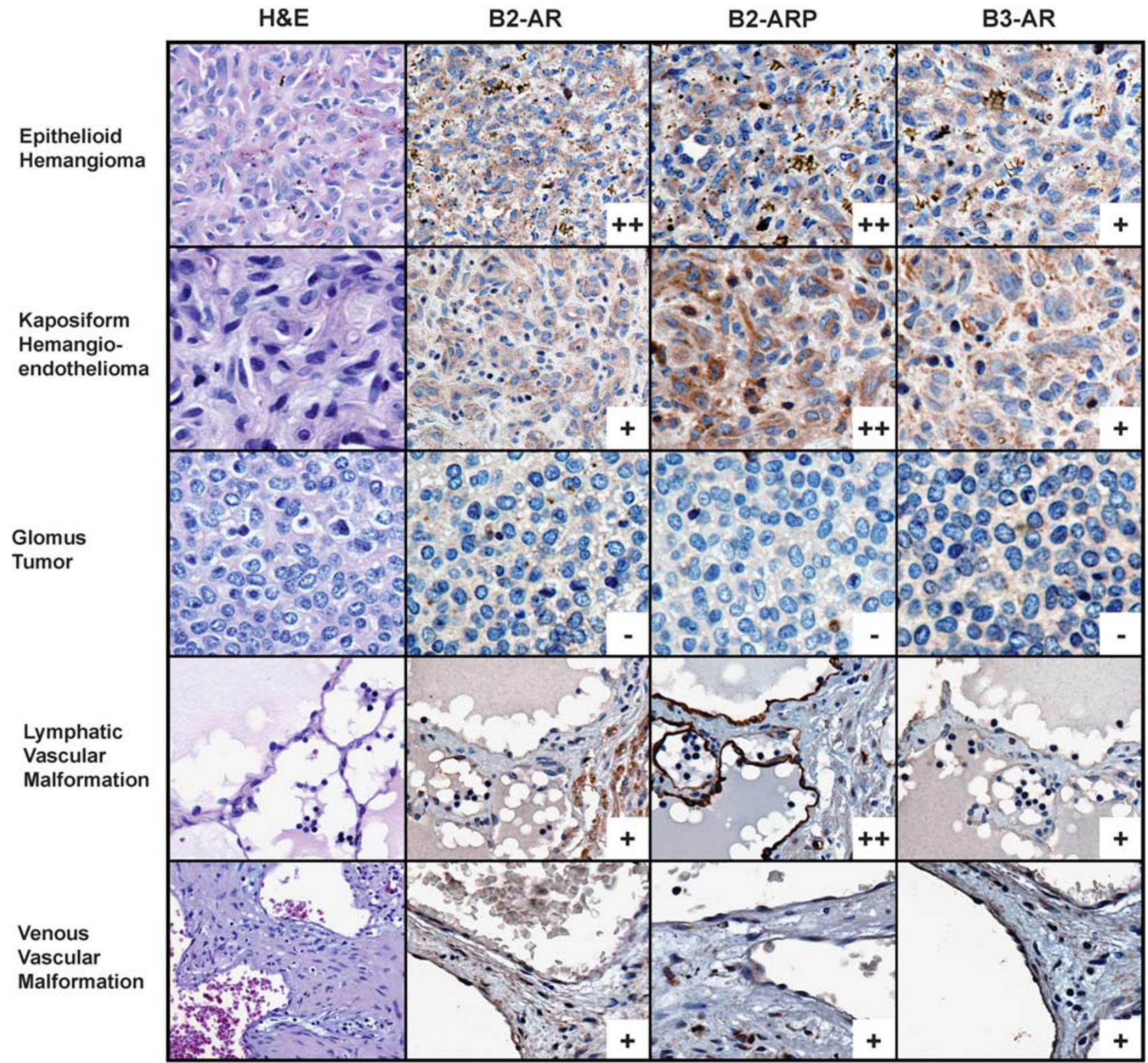

Figure 2 Examples of $\beta$-adrenergic receptor (AR) staining in various vascular lesions. Hematoxylin and eosin staining of cases of epithelioid hemangioma, kaposiform hemangioendothelioma, glomus tumor, lymphatic vascular malformation, and venous vascular malformation was performed, followed by immunohistochemical evaluation with $\beta 2$-AR (B2-AR), its phosphorylated form (B2-ARP), and B3-AR (B3-AR). As shown, epithelioid hemangioma had strong (+ + ) expression of B2-AR and B2-ARP, and weak ( +) expression of B3AR. Kaposiform hemangioendothelioma had strong expression of B2-ARP and weak expression of both B2-AR and B3-AR. Glomus tumor lacked expression of all three receptors. Lymphatic vascular malformation had strong expression of B2-ARP, and weak expression of B2AR and B3-AR. Last, venous vascular malformation had weak expression of all three receptors.

mixed small and large vessel vascular malformation, and intramuscular vascular malformation, and in the majority of epithelioid hemangiomas.

Most tumors had at least weak expression of all $\beta$ ARs (data not shown). However, B2-AR was only expressed in 33 of 44 angiosarcomas $(77 \%), 1$ of 3 hemangiopericytomas (33\%), 2 of 4 littoral cell angiomas (50\%), and 3 of 5 glomus tumors (60\%). B2-AR was not expressed in the one case of hobnail hemangioendothelioma and the one case of retiform hemangioendothelioma. B2-ARP was only expressed in 32 of 44 angiosarcomas $(73 \%), 3$ of 5
Kaposi sarcomas (60\%), 1 of 2 pyogenic granulomas $(50 \%), 3$ of 5 glomus tumors $(60 \%)$, and 1 of 2 intramuscular vascular malformations (50\%). B3-AR was only expressed in 1 of 2 kaposiform hemangioendotheliomas $(50 \%), 25$ of 38 angiosarcomas (66\%), 2 of 4 intimal sarcomas (50), 1 of 5 littoral cell angiomas $(20 \%)$, and 2 of 5 glomus tumors (40\%); B3-AR was not expressed in the hobnail hemangioendothelioma lesion.

Strong expression of B2-AR and B2-ARP were not necessarily congruent, but weak expression of the counterpart was usually present. 
Table $1 \beta$-Adrenergic strong expression in vascular lesions

\begin{tabular}{|c|c|c|c|c|c|c|}
\hline \multirow[t]{2}{*}{ Vascular lesion } & \multicolumn{2}{|c|}{$B 2-A R$} & \multicolumn{2}{|c|}{$B 2-A R P$} & \multicolumn{2}{|c|}{$B 3-A R$} \\
\hline & $\mathbf{N}^{\mathrm{a}}$ & $\%^{\mathrm{b}}$ & $\mathbf{N}^{\mathrm{a}}$ & $\%^{\mathrm{b}}$ & $\mathbf{N}^{\mathrm{a}}$ & $\%^{\mathrm{b}}$ \\
\hline Infantile hemangioma & 5 & 100 & 5 & 80 & 4 & 100 \\
\hline Epithelioid hemangioma & 4 & 50 & 4 & 100 & 4 & 75 \\
\hline Spindle cell hemangioma & 2 & 100 & 4 & 50 & 3 & 67 \\
\hline Bone hemangioma & 1 & 100 & 2 & 50 & 1 & 100 \\
\hline Epithelioid hemangioendothelioma & 14 & 50 & 14 & 87 & 13 & 69 \\
\hline Kaposiform hemangioendothelioma & 3 & 67 & 4 & 75 & 2 & 50 \\
\hline Bone hemangioendothelioma & 1 & 100 & 1 & 0 & 1 & 0 \\
\hline Hobnail hemangioendothelioma & 1 & 0 & 1 & 0 & 1 & 0 \\
\hline Retiform hemangioendothelioma & 1 & 0 & 1 & 100 & 1 & 0 \\
\hline Angiosarcoma & 44 & 41 & 44 & 48 & 38 & 21 \\
\hline Intimal sarcoma & 4 & 100 & 4 & 50 & 4 & 50 \\
\hline Kaposi sarcoma & 4 & 75 & 5 & 60 & 3 & 33 \\
\hline Hemangiopericytoma & 3 & 33 & 3 & 67 & 3 & 33 \\
\hline Littoral cell angioma & 4 & 25 & 6 & 83 & 5 & 20 \\
\hline Pyogenic granuloma & 2 & 50 & 2 & 50 & 1 & 0 \\
\hline Glomus tumor & 5 & 40 & 5 & 40 & 5 & 20 \\
\hline Masson’s & 1 & 0 & 2 & 100 & 2 & 100 \\
\hline Vascular malformation, venou & 7 & 57 & 7 & 71 & 6 & 33 \\
\hline Vascular malformation, lymphatic & 4 & 50 & 7 & 57 & 3 & 33 \\
\hline $\begin{array}{l}\text { Vascular malformation, mixed } \\
\text { small and large vessel }\end{array}$ & 1 & 100 & 1 & 100 & 1 & 100 \\
\hline $\begin{array}{l}\text { Vascular malformation, not } \\
\text { otherwise specified }\end{array}$ & 1 & 100 & 1 & 100 & 1 & 0 \\
\hline Intramuscular vascular malformation & 1 & 100 & 2 & 50 & 1 & 100 \\
\hline Lymphangioleiomyomatosis & 1 & 0 & 1 & 100 & 1 & 0 \\
\hline Reactive vascular proliferation & 0 & n.a. & 1 & 0 & 1 & 0 \\
\hline
\end{tabular}

Abbreviations: AR, adrenergic receptor; B2-AR, $\beta 2$-AR; B2-ARP, phosphorylated form of $\beta 2$-AR; B3-AR, $\beta 3$-AR.

${ }^{\mathrm{a}}$ Number of lesions with interpretable staining.

${ }^{b}$ Percent of lesions with strong expression of $\beta$-ARs out of total number of lesions with interpretable staining.

\section{Discussion}

This study is the first to report $\beta$-AR expression in a variety of vascular lesions, including infantile hemangiomas. Proangiogenic factors, such as the VEGF and bFGF are abundant in the proliferative phase of infantile hemangioma, ${ }^{26}$ causing a proliferation of endothelial cells. As shown in this study, B2-AR and B3-AR are both strongly expressed in the (early) proliferative phase of infantile hemangioma, whereas B2-ARP is only weakly expressed. The significance of this observation is unclear, but may be related to the proliferative activity of the lesion, or availability of PKA in the early proliferative phase of infantile hemangiomas compared with the early involutional phase.

The success of propranolol therapy for infantile hemangiomas has lead to consideration of other vascular lesions for treatment. Kaposiform hemangioendotheliomas are aggressive vascular tumors with a high mortality rate, and only variably successful therapeutic strategies. Though only $67 \%$ of kaposiform hemangioendotheliomas expressed B2-AR in this study, Herman et $a l^{27}$ recently reported a 6-year-old child with kaposiform hemangioendothelioma and Kasabach-Merritt syndrome, who was treated with propranolol and 4 weeks of vincristine (compared with the average of 22 weeks of vincristine), resulting in remission for over a year. In addition, propranolol has recently been reported to induce resolution of an aggressive, recurring orbital epithelioid hemangioma. ${ }^{28}$ These examples illustrates that propranolol may be an important single or adjunctive therapy in many vascular lesions.

Though immunohistochemical studies demonstrate B2-AR staining in many lesions, this expression does not indicate that the lesions are responsive to $\beta 2$-receptor antagonists. In this study, $50 \%$ of pyogenic granulomas had strong expression of B2AR. Anecdotally, a patient with a diagnosis of infantile hemangioma was treated with propranolol. This individual failed treatment, and on pathology review, the lesion was found to be a pyogenic granuloma, not an infantile hemangioma. ${ }^{29}$ However, $\beta$-AR expression was not determined in this case, so it is not known whether the pyogenic granuloma did not respond to propranolol due to lack of receptor expression.

We demonstrated variable $\beta$-AR expression in a broad spectrum of vascular lesions. Hence, propranolol or $\beta 3$-receptor antagonists may be of benefit in some of the lesions. $\beta 2$-receptor antagonists such as propranolol could affect apoptosis and decrease the responsiveness to VEGF. Even if they do not completely cure some lesions, $\beta 2$ - or $\beta 3$-receptor antagonists may be able to stop growth or shrink certain lesions, and thereby facilitate surgical resection or other ablative methods. A large clinical trial of vascular tumors would be necessary to determine the significance of immunohistochemical expression of $\beta$-ARs as a biomarker predicting response to propranolol. Such a trial would allow reproducible, quantitative thresholds to be set similar to those used for epidermal growth factor receptor expression in lung cancer therapy. ${ }^{30}$

\section{Acknowledgement}

We thank Dr Brian Rubin, Cleveland Clinic Department of Anatomic Pathology, for his collaboration with Stanford in constructing the vascular tumor tissue microarray.

\section{Disclosure/conflict of interest}

The authors declare no conflicts of interest.

\section{References}

1 Bruckner AL, Frieden IJ. Hemangiomas of infancy. J Am Acad Dermatol 2003;48:477-493 quiz 494-6.

2 Bennett ML, Fleischer Jr AB, Chamlin SL, et al. Oral corticosteroid use is effective for cutaneous hemangiomas: an evidence-based evaluation. Arch Dermatol 2001;137:1208-1213. 
3 Ezekowitz RA, Mulliken JB, Folkman J. Interferon alfa2a therapy for life-threatening hemangiomas of infancy. N Engl J Med 1992;326:1456-1463.

4 Enjolras O, Breviere GM, Roger G, et al. Vincristine treatment for function- and life-threatening infantile hemangioma. Arch Pediatr 2004;11:99-107.

5 Perez Payarols J, Pardo Masferrer J, Gomez Bellvert C. Treatment of life-threatening infantile hemangiomas with vincristine. N Engl J Med 1995;333:69.

6 Moore J, Lee M, Garzon M, et al. Effective therapy of a vascular tumor of infancy with vincristine. J Pediatr Surg 2001;36:1273-1276.

7 Boon LM, MacDonald DM, Mulliken JB. Complications of systemic corticosteroid therapy for problematic hemangioma. Plast Reconstr Surg 1999;104:1616-1623.

8 Dubois J, Hershon L, Carmant L, et al. Toxicity profile of interferon alfa-2b in children: a prospective evaluation. J Pediatr 1999;135:782-785.

9 Barlow CF, Priebe CJ, Mulliken JB, et al. Spastic diplegia as a complication of interferon Alfa-2a treatment of hemangiomas of infancy. J Pediatr 1998; 132:527-530.

10 Leaute-Labreze C, Dumas de la Roque E, Hubiche T, et al. Propranolol for severe hemangiomas of infancy. $\mathrm{N}$ Engl J Med 2008;358:2649-2651.

11 Bigorre M, Van Kien AK, Valette H. Beta-blocking agent for treatment of infantile hemangioma. Plast Reconstr Surg 2009;123:195e-196e.

12 Sans V, de la Roque ED, Berge J, et al. Propranolol for severe infantile hemangiomas: follow-up report. Pediatrics 2009;124:e423-e431.

13 Buckmiller LM. Propranolol treatment for infantile hemangiomas. Curr Opin Otolaryngol Head Neck Surg 2009;17:458-459.

14 Holmes WJ, Mishra A, Gorst C, et al. Propranolol as first-line treatment for infantile hemangiomas. Plast Reconstr Surg 2010;125:420-421.

15 Fulkerson DH, Agim NG, Al-Shamy G, et al. Emergent medical and surgical management of mediastinal infantile hemangioma with symptomatic spinal cord compression: case report and literature review. Childs Nerv Syst 2010;26:1799-1805.

16 Buckmiller LM, Munson PD, Dyamenahalli U, et al. Propranolol for infantile hemangiomas: early experience at a tertiary vascular anomalies center. Laryngoscope 2010;120:676-681.

17 Truong MT, Chang KW, Berk DR, et al. Propranolol for the treatment of a life-threatening subglottic and mediastinal infantile hemangioma. J Pediatr 2010;156: 335-338.

18 Guimaraes S, Moura D. Vascular adrenoceptors: an update. Pharmacol Rev 2001;53:319-356.

19 D'Angelo G, Lee H, Weiner RI. cAMP-dependent protein kinase inhibits the mitogenic action of vascular endothelial growth factor and fibroblast growth factor in capillary endothelial cells by blocking Raf activation. J Cell Biochem 1997;67:353-366.

20 Storch CH, Hoeger PH. Propranolol for infantile haemangiomas: insights into the molecular mechanisms of action. Br J Dermatol 2010;163:269-274.

21 Iaccarino $\mathrm{G}$, Ciccarelli M, Sorriento D, et al. Ischemic neoangiogenesis enhanced by beta2-adrenergic receptor overexpression: a novel role for the endothelial adrenergic system. Circ Res 2005;97:1182-1189.

22 Summers RJ, Kompa A, Roberts SJ. Beta-adrenoceptor subtypes and their desensitization mechanisms. J Auton Pharmacol 1997;17:331-343.

23 Rozec B, Gauthier C. beta3-adrenoceptors in the cardiovascular system: putative roles in human pathologies. Pharmacol Ther 2006;111:652-673.

24 Wallukat G. The beta-adrenergic receptors. Herz 2002;27:683-690.

25 Enjolras O, Mulliken JB. Vascular tumors and vascular malformations (new issues). Adv Dermatol 1997;13: $375-423$.

26 Takahashi K, Mulliken JB, Kozakewich HPW, et al. Cellular markers that distinguish the phases of hemangioma during infancy and childhood. J Clin Invest 1994;93:2357-2364.

27 Hermans DJJ, van Beynum IM, van der Vijver RJ, et al. Kaposiform hemangioendothelioma with KasabachMerritt syndrome: a new indication for propranolol treatment. J Pediatr Hematol Oncol 2011;33:e171-e173.

28 Moss HB, Sines DT, Blatt J, et al. Epithelioid hemangioma responsive to oral propranolol. Ophthal Plast Reconstr Surg 2011; e-pub ahead of print.

29 Truong MT, Perkins JA, Messner AH, et al. Propranolol for the treatment of airway hemangiomas: a case series and treatment algorithm. Int J Pediatr Otorhinolaryngol 2010;74:1043-1048.

30 Pirker R, Pereira JR, von Pawel J, et al. EGFR expression as a predictor of survival for first-line chemotherapy plus cetuximab in patients with advanced non-small-cell lung cancer: analysis of data from the phase 3 FLEX study. Lancet Oncol 2012; $13: 33-42$. 